\title{
Le rôle de l'optométriste dans une approche multidisciplinaire de réadaptation pour la clientèle aînée atteinte d'hémianopsie homonyme : un cas clinique
}

V. Moore, OD

R. Dufour, OD, M.Sc.

A. Mailhot, M.Sc.

M. Carignan, M.Sc.

Vincent Moore OD

Institut Nazareth \& Louis-Braille

1111, rue Saint-Charles Ouest

Longueuil, Québec

J4K 5G4

vincent.moore.inlb@ssss.gouv.qc.ca.
Résumé

L'hémianopsie homonyme se définit comme une perte congruente d'une moitié du champ visuel affectant les deux yeux. Elle est attribuable à une lésion cérébrale post-chiasmatique. Cette analyse de cas unique montre que l'utilisation de différentes aides optiques (lentilles de Peli, lunettes de lecture et loupes) et l'entraînement au balayage visuel permettent d'améliorer grandement l'autonomie et la qualité de vie d'un usager ayant une hémianopsie. Par souci d'efficience, la réadaptation fonctionnelle des défauts de champs visuels chez les aînés aurait avantage à se faire au sein d'une équipe multidisciplinaire qui inclut un optométriste.

\section{Abstract}

Homonymous hemianopia is defined as a congruent loss of half of the field of view in both eyes and is caused by a post-chiasmatic cerebral lesion. This single case report shows that the use of different optical aids (Peli lenses, reading glasses and magnifiers) in combination with a visual scanning training, can improve the autonomy and quality of life of a user suffering from hemianopia. The functional rehabilitation of visual fields defects in the elderly population may be more efficiently managed by a multidisciplinary team that includes an optometrist. 


\section{INTRODUCTION}

L'hémianopsie homonyme $(\mathrm{HH})$ se définit comme une perte congruente d'une moitié du champ visuel affectant les deux yeux. Elle est attribuable à une lésion post-chiasmatique. Elle peut être causée, entre autres, par une ischémie, une hémorragie, un traumatisme, une inflammation ou une néoplasie'. Aux États-Unis, la prévalence de l'HH parmi les individus de plus de 49 ans est estimée à $0,8 \%$. La cause principale de l'hémianopsie est l'accident vasculaire cérébral (AVC). En effet, parmi les patients ayant subi un AVC, $30 \%$ d'entre eux auraient une hémianopsie homonyme ${ }^{3,4}$. Selon la Fondation des maladies du cour du Canada, environ 50000 AVC se produisent chaque année au $\mathrm{Canada}^{5}$, ce qui permet d'estimer à 15500 le nombre de nouveaux cas d'HH post-AVC. La perte de l'hémi-champ visuel est particulièrement dérangeante d'un point de vue fonctionnel, causant des difficultés importantes pour l'exploration visuelle, les déplacements et la lecture, ce qui nuit à l'autonomie de l'individu'. L'étiologie de l'HH amène souvent d'autres déficits moteurs, sensitifs et/ou cognitifs qui peuvent influencer le pronostic fonctionnel. Lorsque l'HH survient chez une personne âgée, elle peut aussi souvent coexister avec d'autres problèmes visuels (cataractes, glaucome, DMLA, etc...) qui compliquent le processus de réadaptation.

Il n'existe pas à ce jour de traitement clairement établi ni de prise en charge consensuelle pour $l^{\prime} H^{6}$. Plusieurs avenues ont été explorées dans le but de limiter les situations de handicap en lien avec l'hémianopsie. Les traitements possibles peuvent être divisés en quatre catégories : l'approche par substitution (aides optiques), l'approche compensatoire (mouvement oculomoteur, stratégie de balayage), l'approche de restauration (visual restoration therapy, VRT) et la stimulation des capacités de détection dans le champ visuel aveugle (blindsight) ${ }^{1}$. Seules les deux premières ont été utilisées dans le cadre du présent rapport de cas. Parmi les aides optiques, les lentilles de Peli, développés par Eli Peli en $2000^{7}$, sont probablement celles qui présentent le plus d'intérêt à l'heure actuelle ${ }^{8,9,10}$. Elles sont constituées de deux segments de lentilles prismatiques de Fresnel de forte puissance placées en positions supérieure et inférieure sur une des deux lentilles des lunettes de l'usager (Figure 1). Ainsi par un déplacement optique des images du côté de la perte de champ vers le côté sain, ces lentilles permettent d'augmenter le champ visuel de 20 à 30 degrés en latéral, dans deux zones situées en haut et en bas du point de fixation (Figure 2) La portion centrale de la lentille demeure sans prisme afin d'éviter une baisse de l'acuité visuelle et une diplopie centrale ${ }^{78}$. Dans une étude multicentrique menée par Bowers, $74 \%$ des 43 sujets ont continué d'utiliser les prismes de Peli de façon continue 6 semaines après leur installation et $47 \%$ les utilisaient toujours après un $a^{8}$. Une autre étude menée ultérieurement par Bowers avec groupe contrôle a aussi confirmé l'efficacité des prismes de Peli dans l'amélioration des déplacements. En effet, $64 \%$ des participants à l'étude ont affirmé que les prismes étaient plus utiles que le placebo ${ }^{10}$.

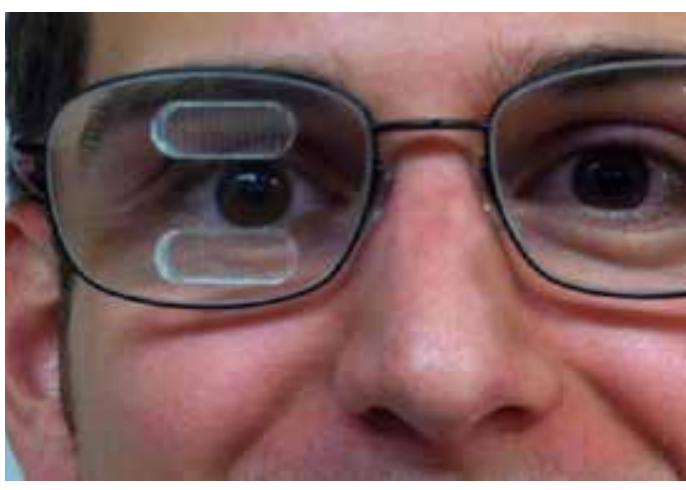

Figure 1. Lentille de Peli positionnée devant OD. Un montage similaire à celui de l'usagère est porté par l'un des auteurs à titre d'exemple.

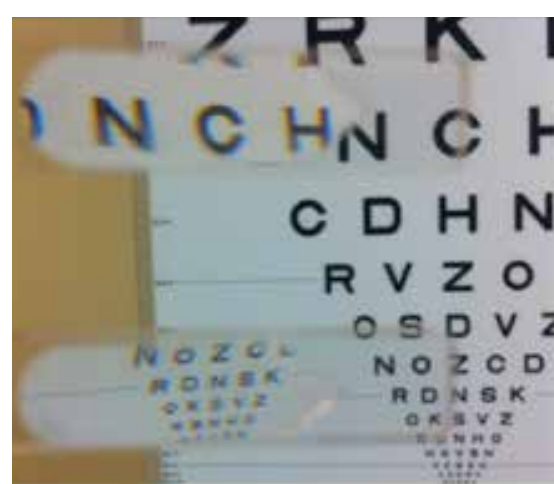

Figure 2. Image vue à travers les lentilles de Peli pour une HH droite. 
Les citoyens du Québec ayant un diagnostic d'hémianopsie complète se qualifient aux services subventionnés par l'État dans les 13 centres de réadaptation en déficience visuelle de la province. Depuis 2011, ils peuvent aussi bénéficier du programme des aides techniques de la Régie de l'assurance-maladie du Québec (RAMQ). Considérant, les résultats intéressants des lentilles de Peli rapportés dans la littérature, une équipe d'un centre de réadaptation s'est questionné sur la façon d'optimiser son offre de service pour cette clientèle, entre autre en y intégrant l'utilisation de cette aide optique. Cette équipe multidisciplinaire était composée d'ergothérapeutes, d'optométristes, de spécialistes en orientation et mobilité (SOM) et de spécialistes en réadaptation en déficience visuelle (SRDV).

Pour bénéficier des services de réadaptation, les individus répondant aux critères d'acuité ou de champ visuel doivent d'abord être référés au centre de réadaptation en déficience visuelle par un optométriste ou un ophtalmologiste. Une évaluation globale des besoins de l'usager est effectuée initialement par un intervenant du centre de réadaptation. Ensuite, l'usager peut être orienté vers d'autres professionnels de l'établissement, car la déficience visuelle exerce des effets sur tous les aspects de la vie. Ainsi, des éducateurs spécialisés, ergothérapeutes, opticiens d'ordonnances, optométristes, psychologues, SOM, SRDV et travailleurs sociaux peuvent collaborer, au besoin, au plan d'intervention de l'usager ${ }^{11}$. Le cas clinique suivant met l'emphase sur le rôle de l'optométriste au sein d'une équipe multidisciplinaire de réadaptation dans les cas de défauts de champs visuels d'origine cérébrale.

\section{MÉTHODE}

L'usagère a subi un examen oculo-visuel complet respectant les standards nord-américains ${ }^{12}$ de pratique au service de basse vision de l'Institut Nazareth et Louis-Braille (INLB), un centre de réadaptation en déficience visuelle. Elle a été par la suite revue pour plusieurs suivis en lien avec la livraison et l'ajustement de différentes aides optiques. Elle avait préalablement accepté de faire partie d'un projet clinique visant à valider un protocole d'ajustement de lentilles de Peli au sein d'une équipe multidisciplinaire de réadaptation et à améliorer les services offerts par l'établissement pour la clientèle aînée atteinte d'HH. Quelques semaines après la fin de la période d'intervention, une collecte de données au dossier clinique de l'usagère a été effectuée avec son autorisation écrite et celle de l'établissement. Toutes les interventions se sont faites en respect de la déclaration d'Helsinki ${ }^{13}$.

\section{CAS CLINIQUE}

Une femme de 72 ans a été référée à l'INLB, sur la recommandation d'un ergothérapeute d'un centre de réadaptation fonctionnelle intensive, pour des situations de handicap en lien avec une $\mathrm{HH}$ droite avec épargne maculaire. L'usagère a été évaluée une première fois par un optométriste du service de basse vision de l'INLB en octobre 2012. À l'histoire de cas, l'usagère affirme avoir subi un AVC en mai 2012. Le rapport d'imagerie cérébrale (effectué le 15 mai 2012) mentionne des lésions touchant la région occipitale et occipito-temporale gauche dans le territoire de l'artère cérébrale postérieure gauche. Le dossier d'hospitalisation indique qu'elle a reçu des services de réadaptation en ergothérapie, en orthophonie et en psychologie pendant trois mois en lien avec des séquelles d'héminégligence droite, d'aphasie anomique légère et de dyslexie modérée, menant à une récupération presque complète de ces fonctions. L'usagère est actuellement sous médication pour le traitement de l'hypertension artérielle, l'ostéoporose, la dyslipidémie et une maladie pulmonaire obstructive chronique. Depuis l'AVC, on lui a aussi prescrit un anticoagulant.

L'usagère explique avoir eu une forte myopie depuis son jeune âge. Suite à l'opération de cataractes subie en 1998, elle se dit moins dépendante de ses lunettes. Elle possède toujours des lunettes à foyers progressifs qu'elle porte en tout temps. Elle n'utilise aucune autre aide optique. Elle rapporte des situations de handicap en lien avec les déplacements, l'exploration visuelle et la lecture. Le résumé des résultats de l'examen optométrique est reporté au tableau 1. Avec les lunettes actuelles, l'usagère a une acuité visuelle au près de $0.8 \mathrm{M}$ à $40 \mathrm{~cm}$ (Light House International near acuity test, New York, NY) et une sensibilité aux contrastes évaluée de façon binoculaire en vision de près de $1.44 \log$ CS (Mars Perceptrix Corporation, Chappaqua, NY) correspondant à une perte modérée. Sa vitesse de lecture est d'environ $60 \mathrm{mots} / \mathrm{min}$. (Minnesota low-vision reading test, version française, INLB, Longueuil, QC) ${ }^{14}$. 
Tableau 1. Examen optométrique initial octobre 2012

\begin{tabular}{|c|c|c|}
\hline \multirow{2}{*}{ Correction portée } & OD & $-0.75 /-0.50 \times 108 \mathrm{add}+2.50$ \\
\hline & OS & $-1.25 /-1.00 \times 033$ add +2.50 \\
\hline \multirow{2}{*}{ Réflexes pupillaires } & OD & 3 mm R2+Lt MG- \\
\hline & OS & 3 mm R2+Lt MG- \\
\hline \multirow{2}{*}{ Motilités oculaires } & OD & Souples et complètes \\
\hline & OS & Souples et complètes \\
\hline \multirow{2}{*}{ Réfraction/ Acuité visuelle } & OD & $-0.50 /-1.00 \times 110(6 / 12)$ \\
\hline & OS & $-1.50 /-1.00 \times 030(6 / 7.5)$ \\
\hline Sensibilité aux contrastes & OU & $1.44 \log \mathrm{CS}$ \\
\hline \multirow{2}{*}{ Test écran } & VL & Orthophore \\
\hline & VP & Orthophore \\
\hline Pression intra-oculaire & OD & $16 \mathrm{~mm} \mathrm{Hg}$ \\
\hline (Goldmann@11h) & OS & $16 \mathrm{~mm} \mathrm{Hg}$ \\
\hline Champs visuels & OD & $\begin{array}{l}\text { Hémianopsie droite complète avec épargne maculaire } \\
\text { et une constriction importante du champ résiduel } \\
\text { gauche. }\end{array}$ \\
\hline $\begin{array}{l}\text { (Octopus 900, Haag-Streit, } \\
\text { méthode dynamique, cible } \\
\text { III } 4 \text { e, } 5 \mathrm{deg} / \mathrm{s} \text { ) }\end{array}$ & OS & $\begin{array}{l}\text { Hémianopsie droite complète avec épargne } \\
\text { maculaire et perte en position supérieure liée à un } \\
\text { blépharochalasis. }\end{array}$ \\
\hline
\end{tabular}

Tableau 2. Aides visuelles recommandées

\begin{tabular}{|l|l|}
\hline \multicolumn{1}{|c|}{ Aides visuelles } & \multicolumn{1}{c|}{ Rendement } \\
\hline Bilentilles (progressives) Add +300 & $\begin{array}{l}\text { lit } 0.8 \mathrm{M} \text { à } 30 \mathrm{~cm} \text { (Light House International near } \\
\text { acuity test) }\end{array}$ \\
\hline $\begin{array}{l}\text { Lunettes de lecture }+4.00 \text { avec prismes } \\
\text { jougués 5 d.p. posés à droite }\end{array}$ & $\begin{array}{l}\text { lit } 80 \text { mots/min (Minnesota low-vision reading } \\
\text { test, version française, INLB). }\end{array}$ \\
\hline Loupe dôme $80 \mathrm{~mm} 2 \mathrm{x}$ & $\begin{array}{l}\text { lit } 0.6 \mathrm{M} \text { à } 30 \mathrm{~cm} \text { (Light House International near } \\
\text { acuity test) }\end{array}$ \\
\hline Filtre solaire de type Fit over ambre 15\% & $\begin{array}{l}\text { Augmentation du confort visuel à l'extérieur, } \\
\text { diminution des plaintes d'éblouissement }\end{array}$ \\
\hline $\begin{array}{l}\text { Lampe d'appoint Ott Lite (ampoule } \\
\text { fluorescente } 13 \mathrm{~W} \text { qui simule le spectre de } \\
\text { la lumière du jour) (Ott Lite Technology, } \\
\text { Tampa, USA) }\end{array}$ & $\begin{array}{l}\text { Diminution de la fatigabilité en lecture par une } \\
\text { augmentation des contrastes et une réduction de } \\
\text { l'éblouissement }\end{array}$ \\
\hline Canne de détection longue & $\begin{array}{l}\text { Amélioration de la posture et augmentation de la } \\
\text { vitesse de marche en permettant la détection des } \\
\text { obstacles en position inférieure }\end{array}$ \\
\hline
\end{tabular}


L'examen au biomicroscope montre une ptose bilatérale liée à un blépharochalasis, des cornées claires et des lentilles intra-oculaires en chambre postérieure claires et bien centrées. L'examen du fond d'œil sous dilatation révèle des vitrés antérieurs clairs, des nerfs optiques présentant une insertion oblique, des croissants scléraux importants et des excavations papillaires centrales (rapport $\mathrm{C} / \mathrm{D}=0.2 / 0.2 \mathrm{OU}$ ). De plus, une zone d'atrophie chori-rétinienne circulaire est observée en zone paramaculaire supérieure OD. Finalement, l'examen de la rétine périphérique montre des zones étendues de dégénérescence de types pavimenteuses et palissadiques. Ces dernières observations sont compatibles avec un diagnostic de myopie dégénérative ${ }^{15}$. De plus, on note la présence d'une membrane épirétinienne (MER) inféro-nasale à la macula OD d'une taille d'environ un diamètre de disque optique.

Suite à des évaluations en salle d'examen par l'optométriste et dans le milieu de vie par l'équipe de réadaptation, certaines aides visuelles sont recommandées à l'usagère (tableau 2). Par la suite, des entraînements à domicile avec un SRDV sont effectués pour développer des stratégies compensatoires et faciliter l'utilisation des aides visuelles dans le cadre des activités de la vie quotidienne. De plus, suite à une évaluation par un SOM, un plan d'intervention est entamé afin d'augmenter la sécurité, l'aisance ainsi que l'autonomie dans les déplacements extérieurs, tout en développant les habiletés de balayage visuel (approche compensatoire).

Un premier suivi optométrique est prévu en janvier 2013. Le champ visuel est refait. En effet, une récupération spontanée du champ visuel est souvent observée dans les cas d'hémianopsie récente $^{16}$. Les dimensions du champ visuel sont stables. Malgré les entraînements et les aides visuelles reçus précédemment, l'usagère rapporte encore des situations de handicap en lecture ainsi que lors de ses déplacements dans les lieux publics achalandés. Elle ne se sent pas en sécurité dans ses déplacements extérieurs et elle rapporte une anxiété suffisante pour nécessiter un accompagnement du conjoint en tout temps. Elle omet occasionnellement les objets situés dans son champ visuel droit lors de son exploration visuelle et se fatigue rapidement en lecture au point de ne faire que de la lecture de décodage. L'essai de prismes de Peli lui est alors proposé. Toutefois, compte tenu de ses autres problèmes de santé et de ses difficultés à sortir à l'extérieur par temps froid, elle préfère attendre quelques semaines. Il est à noter qu'au moment des entraînements, l'usagère vivait aussi des difficultés d'adaptations psychosociales en lien avec sa déficience visuelle qui ont pu influencer le déroulement de la période de réadaptation. Une référence en ergothérapie est faite à ce moment par l'optométriste, dans le but de soutenir l'équipe de réadaptation dans l'analyse des problématiques perceptuelles et cognitives qui sont fréquentes avec un diagnostic d'hémianopsie et qui peuvent limiter le rendement dans la vie quotidienne. L'ergothérapeute confirme que l'état de l'usagère se situe dans les normes attendues en fonction de l'âge au niveau cognitif et perceptuel (incluant l'héminégligence) lors de l'évaluation fonctionnelle et lorsqu'objectivé avec des outils de dépistage standardisés (Motor-free visual perception test - MVPT- $3^{17}$; Occupational Therapy Adult Perceptual Screening Test - OT-APST ${ }^{18}$; Montreal Cognitive Assessment, - MoCA ${ }^{19}$ ). Il est donc conclu que les situations de handicap observées sont surtout liées à l'HH.

L'usagère est revue en clinique de basse vision en juin 2013. L'essai des prismes de Peli a lieu à cette occasion après avoir confirmé la motivation de l'usagère. Une monture est sélectionnée par l'opticienne d'ordonnances pour la pose ultérieure des prismes de Peli. Même si certains auteurs ont affirmé que les prismes pouvaient être installés sur des lunettes à double-foyers ${ }^{8,20}$, une lentille porteuse unifocale est commandée afin de faciliter l'adaptation. Une monture avec des plaquettes de nez ajustables et une grande dimension verticale (cote $B=39.5 \mathrm{~mm}$ ) est retenue pour répondre aux suggestions du fabricant qui recommande un minimum de $35 \mathrm{~mm}$ pour la pose des prismes ${ }^{21}$. Afin de déterminer l'œil sur lequel les prismes seront installés, quelques tests supplémentaires concernant la vision binoculaire sont effectués. La vérification de la dominance oculaire avec embrouillement convexe montre une dominance de OS. Il y a fusion des images au filtre rouge à $40 \mathrm{~cm}$ et $6 \mathrm{~m}$. Aucune suppression n'est notée au test de "Worth 4 -dots" à $40 \mathrm{~cm}$. La décision est alors prise d'installer le prisme sur OD malgré une dominance OS afin de maximiser le gain de champ visuel à droite. Ross et collaborateurs ont d'ailleurs démontré que dans le cas d'une vision binoculaire normale le taux de détection des stimuli à 
travers les prismes est équivalent, peu importe l'oeil sur lequel les prismes sont installés ${ }^{22}$. Par contre, ces auteurs soulignent que chez des usagers ayant une dominance oculaire marquée (vision binoculaire anormale), les taux de détection seraient inférieurs lorsque les prismes sont placés devant l'œil non dominant. Ainsi à la livraison des lunettes, un premier prisme de Fresnel sectoriel temporaire de 40 d.p. horizontal (Chadwick Optical Inc., Souderton, PA) est installé en position supérieure. Une première séance d'entrainement aux prismes est prévue la journée même de la livraison avec le SOM. Une autre séance dans l'environnement immédiat de l'usagère est prévue avant le prochain suivi en optométrie. Puisque l'usagère doit gérer maintenant trois lunettes différentes, un rappel de l'utilisation à faire avec chacune de celles-ci lui a été fait: les lunettes avec prismes sectoriels doivent être portées seulement lors des déplacements piétons à l'extérieur, les lunettes de lecture doivent être portées seulement pour les tâches nécessitant une vision précise à $25 \mathrm{~cm}$ (lecture, travaux manuels) et les lunettes à foyers progressifs doivent être portées pour toutes les autres activités, c'est-à-dire celles nécessitant l'utilisation de la vision intermédiaire (ordinateur) et lorsque l'usagère doit utiliser simultanément la vision de loin et de près.

Deux semaines plus tard, l'usagère est revue en optométrie. Elle rapporte peu de difficultés avec le prisme en position supérieure, mais aussi peu de bénéfices. La hauteur du prisme est donc diminuée, compte tenu de la ptose, afin de maximiser le gain de champ. Le deuxième prisme de 40 d.p. est alors installé en position inférieure avec une distance interprisme de 10,5 mm. L'acuité étant légèrement diminuée sur l'oeil droit, une évaluation du fond d'oeil est effectuée et permet de constater une progression de la MER. Une référence à un rétinologue est alors proposée à l'usagère pour une opinion sur la possibilité d'un traitement. Finalement, à la fin de cette quatrième visite, l'usagère est accompagnée à l'extérieur par le SOM pour la poursuite des entraînements.

L'usagère est revue en optométrie un mois plus tard. Un champ visuel binoculaire effectué avec les prismes confirme alors une augmentation de champ visuel d'environ 20 degrés en latéral autant en supérieur qu'en inférieur du point de fixation. L'évaluation du SOM démontre une amélioration du port de la tête car l'usagère applique maintenant un balayage visuel efficace. On observe également une augmentation de la vitesse de marche et l'usagère exprime une augmentation du sentiment de confiance à exécuter ses déplacements, et ce, même en milieu non-familier. Toutefois, puisque l'usagère se plaint que le rebord du prisme supérieur nuit en position primaire de regard (elle doit relever le menton) les deux prismes sont déplacés de $1 \mathrm{~mm}$ vers le haut de la lentille porteuse. Une commande est alors faite pour des prismes surfacés en PMMA de 57 d.p. horizontaux montés selon les nouvelles mesures. Cette puissance de prisme est disponible seulement dans la version permanente et devrait permettre d'augmenter le gain de champ visuel ${ }^{8,9}$.

En octobre 2013, l'usagère est revue de nouveau en clinique de basse vision pour la livraison des lunettes avec prismes permanents. Un champ visuel binoculaire est refait et confirme une augmentation d'environ 30 degrés en latéral, en supérieur et inférieur du point de fixation, par rapport à l'examen initial. Un dernier entraînement est aussi effectué avec le SOM pour s'assurer de la sécurité des déplacements piétonniers avec les prismes de plus forte puissance. Le rapport de l'ophtalmologiste consultant est aussi reçu. Celui-ci confirme la présence d'une MER après imagerie de la rétine (OCT, tomographie par cohérence optique) et suggère une chirurgie. Toutefois, l'usagère préfère attendre.

Un mois après la livraison, un questionnaire téléphonique est administré par une opticienne afin d'évaluer le niveau de satisfaction de l'usagère aux lentilles de Peli. Elle affirme alors porter les prismes en moyenne 7 heures par jour. Ceux-ci seraient très utiles, selon elle, pour éviter les obstacles lors des déplacements à l'extérieur. Elle prévoit continuer à les utiliser dans le futur. Elle est très heureuse et accepte volontiers que l'on partage son expérience positive avec nos collègues. 


\section{DISCUSSION}

L'optométriste a donc un rôle significatif à jouer dans la réadaptation des usagers atteints d'HH. La réussite de l'ajustement des prismes de Peli peut être influencée par plusieurs facteurs. En effet, puisque l'HH touche davantage les individus âgés, les professionnels de la vision qui souhaitent prendre en charge cette clientèle ont souvent à tenir compte des effets d'autres pathologies concomitantes (oculaires ou autres). Dans le cas présent, l'usagère présentait une rétinopathie myopique, une membrane épirétinienne et un blépharochalasis affectant l'acuité visuelle et les champs visuels. Ces conditions sont susceptibles d'influencer le pronostic visuel à moyen et long terme, et ce, indépendamment du succès des aides optiques proposées pour l'HH. Les articles publiés sur l'efficacité des prismes de Peli font peu mention des pathologies concomitantes ou rapportent le cas d'usagers sans autres pathologies ou avec une bonne acuité visuelle ${ }^{7,8,9,10}$. Ceci ne représente pas la majorité de la clientèle en centre de réadaptation. De plus, et même si notre usagère en était exempte, il est important de considérer les autres séquelles cognitives, perceptuelles ou physiques souvent associées à l'étiologie de l'HH (AVC, tumeur, traumatisme). Ces dernières peuvent influencer la réussite de l'adaptation aux aides optiques, incluant les prismes de Peli, d'où la nécessité d'une approche multidisciplinaire pour cette clientèle.

Ce cas clinique met aussi en évidence les nombreuses visites requises et la quantité d'intervenants impliqués pour la réadaptation des usagers âgés atteints d'HH. Toutefois, les bénéfices peuvent être grands au niveau du maintien de l'autonomie et de la prévention des chutes. La déficience visuelle étant l'un des plus importants facteurs de risque de chutes ${ }^{23,24}$, toute intervention permettant de diminuer ce risque de manière significative est souhaitable. Cependant, il est important de considérer que les prismes peuvent aussi engendrer un risque de chute supplémentaire par l'ajout d'information visuelle, d'où l'importance d'une sélection rigoureuse des usagers. Les traitements décrits dans le présent cas clinique combinent deux différentes approches, soit l'approche par substitution (lentilles de Peli) et l'approche compensatoire (entraînements avec SOM, effectués avant et après l'ajout des prismes). D'autres études seraient nécessaires afin de comparer l'efficacité relative de chacune de ces approches auprès de la clientèle aînée, ainsi que l'effet de la combinaison de certaines de ces approches sur la diminution des situations de handicap.

Le protocole d'ajustement utilisé à l'INLB est très semblable à celui décrit par Bowers et $\mathrm{coll}^{8}$. La différence majeure est que nous n'avons pas permis l'utilisation des lentilles à doublefoyers et que l'usagère a bénéficié de plus de temps d'entraînement avec un SOM. Ces deux éléments pourraient avoir augmenté les chances de succès de l'adaptation aux prismes. Aussi, il a été suggéré que les deux prismes temporaires pourraient être posés en même temps pour accélérer l'entraînement avec certains usagers. Toutefois, compte tenu des visites requises pour les ajustements et la prise des mesures, il n'est pas certain que le nombre de visites en optométrie serait ainsi réduit. Il serait intéressant de documenter l'impact d'un protocole accéléré de ce type d'intervention chez la clientèle âgée.

L'usagère a reçu des prismes dont l'orientation était horizontale et non oblique. Or des résultats intéressants ont été obtenus avec des prismes de puissance équivalente mais avec une orientation oblique, qui permettent une augmentation de champ en vision centrale ${ }^{25}$. Il faudrait par contre vérifier si la confusion possiblement associée aux prismes obliques pourrait influencer le succès de l'adaptation, particulièrement auprès de la clientèle aînée. Aussi, malgré le fait que certains auteurs montrent que ces lentilles aident à développer les habilités compensatoires permettant la reprise du permis de conduire ${ }^{26,27}$, des études avec un plus grand nombre d'individus devraient être faites pour conclure à la sécurité de cette aide optique sur la route et déterminer la méthode d'entraînement optimale. Dans le cas présent, il a été recommandé à l'usagère de ne pas conduire avec les lentilles de Peli.

Les usagers des centres de réadaptation en déficience visuelle du Québec ayant un diagnostic d'hémianopsie complète se qualifient maintenant au programme des aides techniques de la RAMQ et les lentilles de Peli sont incluses dans les aides attribuables selon le décret de la 
RAMQ depuis 2013. Compte tenu de la particularité de la clientèle avec $\mathrm{HH}$, le travail en équipe multidisciplinaire permet de cibler plus efficacement tous les besoins de l'usager en utilisant les ressources de différents professionnels. De façon plus spécifique, la contribution des SOM pour l'entraînement des usagers avec prismes de Peli apparaît essentielle. L'importance de leur rôle a d'ailleurs déjà été mentionnée dans la littérature ${ }^{28}$. Ainsi, les auteurs croient que les conditions actuelles du système québécois, où l'usager a accès aux équipes spécialisées des centres de réadaptation ainsi qu'aux aides visuelles requises, sont facilitantes pour les usagers et assureraient peut-être un meilleur succès pour la réadapation de la clientèle avec $\mathrm{HH}$.

\section{CONCLUSION}

Tel que l'illustre ce cas, les lentilles de Peli peuvent améliorer le quotidien d'une usagère atteinte d'HH en augmentant sa confiance dans les déplacements. Par contre, la littérature mentionne que ce type d'aides visuelles ne serait pas utile pour tous les usagers atteints d'HH. D'autres études seraient requises pour établir une méthode plus efficace de sélection des candidats. Les ergothérapeutes sont des alliés importants dans la sélection et l'orientation vers les autres approches selon l'état et les besoins de l'usager ( déficits cognitifs, perceptuels ou moteurs). Finalement, l'apport de l'optométriste est essentiel afin d'établir un plan d'intervention efficient qui considère l'ensemble des approches d'intervention auprès d'un usager cérébrolésé. Il est important que les optométristes interviennent auprès de cette clientèle et qu'ils fassent valoir leur expertise complémentaire au niveau de la santé oculaire et de l'optique ophtalmique

\section{BIBLIOGRAPHIE}

1. Pouget MC, Lévy-Bencheton D, Prost M, Tilikete C, Husain M, Jacquin-Courtois S. (2012), Acquired visual field defects rehabilitation: critical review and perspectives. Ann Phys Rehabil Med; 55(1):53-74.

2. Gilhotra JS, Mitchell P, Healey PR, et al. (2002), Homonymous visual field defects and stroke in older population. Stroke;33:2417-20.

3. Pambakian AL, Kennard C. (1997), Can visual function be restored in patients with homonymous hemianopia? Br J Ophthalmol;81:324-8.

4. Suchoff IB, Kapoor N, Ciuffreda KJ, Rutner D, Han E, Craig S. (2008), The frequency of occurrence, types, and characteristics of visual field defects in acquired brain injury: a retrospective analysis. Optometry;79(5):259-65.

5. Hakim A M, Silver F, Hodgson C, (1998), Organized stroke care: A new era in stroke prevention and treatment, Can Med Assoc J; 159(6): S1.

6. Pollock A, Hazelton C, Henderson CA, Angilley J, Dhillon B, Langhorne P, Livingstone K, Munro FA, Orr $\mathrm{H}$, Rowe FJ, Shahani U. (2011). Interventions for visua field defects in patients with stroke. Cochrane Database Syst Rev (10):CD008388. doi: 10.1002/14651858. CD008388.pub2. Review

7. Peli, E. (2000), Field expansion for homonymous hemianopia by optically induced peripheral exotropia. Optom Vis Sci; 77(9):453-464.

8. Bowers A.R., Keeney K, Peli E,(2008), Communitybased trial of a peripheral prism visual expansion device for hemianopia. Arch Ophthalmol;126(5):657-664.

9. O'Neil EC, Connell PP, O'Connor JC, et al. (2011), Prism therapy and visual rehabilitation in homonymous visual field loss, Optom Vis Sci. ;88(2):263-8.

10. Bowers A.R., Keeney K, Peli E,(2014), Randomized crossover clinical trial of real and sham peripheral prism glasses for hemianopia, JAMA Ophthalmol.;132(2):214-222.

11. Institut Nazareth et Louis-Braille (2013). Les programmes-cadres de l'Institut Nazareth et LouisBraille. Longueuil : Qc.

12. Carlson N.B., Kurtz D,Clinical Procedures for Ocular Examination, third edition. McGraw Hill Professionnal; 2003.
13. Déclaration d'Helsinki de l'Association Médicale Mondiale - Principes éthiques applicables à la recherche médicale impliquant des êtres humains, http://www. wma.net/fr/30publications/10policies/b3/, consultée en ligne le 4 juin 2014

14. Sénécal, M.J., Gresset, J. \& Overbury, O. (2003). Version française du Minnesota Reading Test, L’incapacité visuelle et la réadaptation (Vol. V, pp. 3134). Montréal: Université de Montréal.

15. Kanski, J. J. Acquired macular disorders, In: Kanski, J. J., Clinical Ophthalmology: A Systematic Approach, 6th Edition, Butterworth-Heinemann,2007: 389-437.

16. Zang X, Kedar S. Lynn M.J. et al, (2006) Natural history of homonymous hemianopia, Neurology, 66;903.

17. Colarusso, R.P. \& Hammill,D.D. (2003). Motor free visual perceptual test 3rd edition, examiner's manual, Novato, CA: Academic Therapy Publications, Inc.

18. Cooke, D. M., McKenna, K., Fleming, J. (2005). Development of a standardized occupational therapy screening tool for visual perception in adults. Scand Occup Ther, 12:59-71.

19. Nasreddine, Z.S., Phillips, N.A., Bédirian, V, Charbonneau, S, Whitehead, V, Collin, I, Cummings, J.L., Chertkow, H. (2005).The Montreal Cognitive Assessment (MoCA): A brief screening tool for mild cognitive impairment. J Am Geriatr. Soc. 53:695-699.

20. The Low Vision Center of Indiana. http://www.hemianopsia.net/the-ep-lens-for-hemianopsia/, consulté en ligne le 22 avril 2014.

21. Chadwick Optical Inc, (2010) Peli Lens Fitting kit. http://www.chadwickoptical.com/index_files/pelilensforhemianopia.htm, consulté en ligne le 13 juillet 2014.

22. Ross N.C., Bowers A.R., Peli E.(2012) Peripheral glasses : Effects of dominance, suppression and background. Optom Vis Sci; 89(9):1343-1352.

23. Harwood, R. (2001). Visual problems and falls. Age and Ageing, 30-S4, 13-18. Vu dans Duquette, McClure, Baril (2013). La prévention des chutes chez les aînés ayant une déficience visuelle. Institut Nazareth et Louis-Braille. Récupéré du site de l'éditeur : http:// inlb.qc.ca/librairies/sfv/telecharger.php?fichier=1309 
24. Ivers R.Q., Norton R., et al. (2000) Visual Impairment and risk of hip fracture, Am J Epidemiol 152(7):633639.

25. Bowers AR, Tant M, Peli E. (2012). A pilot evaluation of on-road detection performance by drivers with hemianopia using oblique peripheral prisms. Stroke Res Treat.;2012(3):176806-10.

26. Bowers AR, Ananyev E, Mandel AJ, Goldstein RB, Peli E. (2014). Driving with hemianopia: IV. Head scanning and detection at intersections in a simulator. Invest Ophthalmol Vis Sci.;55(3):1540-8.

27. Moss AM, Harrison AR, Lee MS.(2014). Patients with homonymous hemianopia become visually qualified to drive using novel monocular sector prisms. J Neuroophthalmol.;34(1):53-6.

28. Toffel K, Rumer D, Jun W. (2012) Hemianopsia and falls prevention, Insight : research \& practice in visual impairment \& blindness; 5(1):41-46.

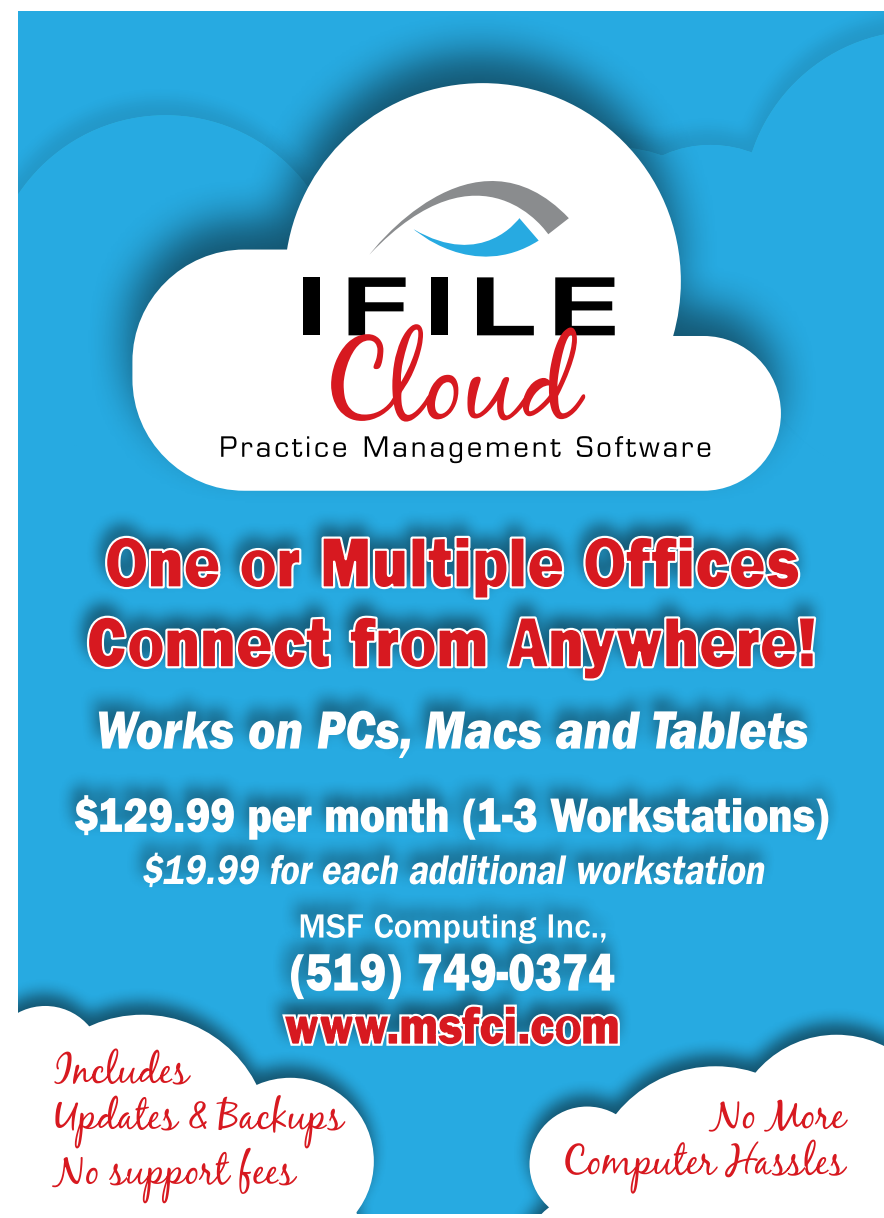

